\title{
La feminización de la lengua francesa y los estudiantes universitarios de FLE
}

\author{
Ana María PÉREz LACARTA \\ Universidad de Valladolid \\ Área de Filología Francesa, Facultad de Traducción e Interpretación \\ amapela@ffr.uva.es
}

Recibido: 08/09/2014

Aceptado: 12/12/2014

\section{Resumen}

En este artículo tratamos de dar respuesta a las preguntas que se plantean en torno a la feminización de la lengua francesa. Examinamos la relación que existe entre género y sexo, las diferentes clases de sexismo, los recursos del francés para formar femeninos, el tipo de obstáculos que encuentra, la posición adoptada por diversos países francófonos. Y, al final del texto, proponemos una serie de actividades de aprendizaje dirigidas a los estudiantes universitarios de FLE con el fin de que puedan resolver por sí mismos las dificultades con que pudieran tropezar en relación con este tema.

Palabras clave: feminización de la lengua francesa, sexismo lingüístico, países francófonos, estudiantes universitarios de FLE, actividades formativas.

\section{La féminisation de la langue française et les étudiants de FLE à l'université}

\section{Résumé}

Dans cet article nous essayons de donner une réponse aux questions qui se posent sur la féminisation de la langue française. On y examine la relation qui existe entre genre et sexe, les différents types de sexisme, les ressources du français pour former des mots féminins, le genre d'obstacles que la langue rencontre, les prises de position adoptées par divers pays francophones. Et, à la fin du texte, nous proposons un ensemble d'activités d'apprentissage adressées aux étudiants de FLE à l'université afin qu'ils deviennent capables de résoudre par eux-mêmes les difficultés qu'ils pourraient trouver à ce sujet.

Mots clés: féminisation de la langue française, sexisme linguistique, pays francophones, étudiants de FLE à l'université, activités d'apprentissage.

\section{The feminization of French language and FFL university students}

\begin{abstract}
This article tries to give an answer to the questions raised about the feminization of the French language. It examines the relationship that exists between gender and sex, the different types of sexism, the resources of the French language to form the feminine, the kind of obstacles it faces, and the stance of the French-speaking countries on the matter. The article ends by proposing a number of learning activities for FFL university students so that they can solve by themselves the learning difficulties they might encounter on the subject.
\end{abstract}


Key words: feminization of the French language, linguistic sexism, French-speaking countries, FFL university students, learning activities.

Sumario: Introducción. 1. Reflexiones sobre la feminización de la lengua francesa. 1.1. Relación entre género y sexo. 1.2. Tipos de sexismo. 1.2.1. Gramatical. 1.2.2. Léxico-semántico. 1.2.3. Discursivo. 1.3. Progresivas modificaciones: diferencias entre países y entre lengua oral y lengua escrita. 1.4. Escollos y soluciones. 1.5. ¿Intervención o evolución lingüística? 2. Actividades formativas. 3. Conclusión.

\section{Referencia normalizada:}

Pérez Lacarta, A. M. (2015) «La feminización de la lengua francesa y los estudiantes universitarios de FLE». Thélème. Revista Complutense de Estudios Franceses, Vol.30, Núm. 1: 115-127. http://dx.doi.org/10.5209/rev_THEL.2015.v30.n1.45629

\section{Introducción}

El interés por la feminización de la lengua francesa y la necesidad de encontrar una respuesta precisa a las dudas planteadas por los estudiantes universitarios de FLE en relación con dicho tema nos han llevado a ahondar en un terreno accidentado, que en las últimas décadas se ha ido nivelando, pero que todavía hay que allanar. Con objeto de facilitar su exploración, vamos a formular varias preguntas que invitan a la reflexión y que, acompañadas de diversas actividades formativas, pueden contribuir a despejar el recorrido. Para avanzar con paso firme, tendremos en cuenta el criterio de diferentes especialistas de la lengua francesa, así como también el parecer de profesionales de otros ámbitos, que se han involucrado en los debates que se han ido abriendo al respecto.

Estimulando la curiosidad por saber si existe relación entre género y sexo en la lengua francesa y si el masculino tiene verdadero carácter genérico; invitando a averiguar si se da la discriminación sexista en este idioma y, en caso afirmativo, si se refleja en la comunicación escrita y hablada y en todos los países francófonos por igual; dirigiendo la atención hacia los obstáculos que impiden la feminización lingüística y hacia los recursos de que dispone el francés para salvarlos; centrando la mirada en profesiones y cargos que anteriormente la mujer no desempeñaba; planteando la conveniencia de modificar la evolución natural del francés a fin de que la igualdad entre sexos se haga realidad y animando a comparar las soluciones que ofrecen otras lenguas a este problema lingüístico lograremos que el estudiante tenga una perspectiva amplia del tema y extraiga sus propias conclusiones. 


\section{Reflexiones sobre la feminización de la lengua francesa}

\subsection{Relación entre género y sexo}

En primer lugar, convendría preguntarse si existe relación entre género y sexo en la lengua francesa y si es pertinente la utilización del masculino genérico. Diversos especialistas en la materia han abordado este tema y han respondido afirmativamente a la primera pregunta: en lo que concierne a las personas, ambas categorías — gramatical y biológica - están estrechamente vinculadas. Le bon usage (1986: 788) dice: “Ce n’est que pour les noms animés que le genre n’est pas arbitraire, parce qu'il est déterminé par le sexe des êtres désignés, du moins pour ce qui concerne, en général, les êtres humains [...]. Pour la majorité des noms d'animaux, en effet, le genre reste sans lien avec le sexe”.

Y señala los casos en los que no se da dicha correspondencia: femeninos que designan a hombres, masculinos que se aplican a mujeres o sustantivos de uno u otro género que se emplean para ambos sexos. Dado que la sociedad está cambiando y que la lengua evoluciona progresivamente, algunas de estas disparidades van desapareciendo con el tiempo; no obstante, otras perduran y en ocasiones dan lugar a frases absurdas. Ajustándose al principio de que el significante y el significado tienen que ser del mismo género y sexo para las personas (Niedzwiecki, 1994: 90), términos como une ordonnance se masculinizan (un ordonnance); o como mannequin, sustantivo masculino que designa desde el siglo XX (Rey, 1998: 2124) a la mujer que exhibe colecciones de alta costura —y, desde 1950, también a los hombres que ejercen dicha profesión-, se feminizan (une mannequin o une mannequine).

En lo que concierne al uso no específico del masculino, Jean Rousseau (1998: 11-12) distingue entre dos tipos de sustantivos: aquellos que, al igual que los femeninos personne, victime, etc., no poseen en francés un equivalente del género opuesto, como, por ejemplo, un individu; y los que sí lo tienen (Les Parisiens, les hommes, etc.). Respecto a los primeros precisa: “[...] ces mots ne peuvent avoir un prédicatif adjectif du genre opposé au leur, car ils imposent un accord strictement syntaxique et non sémantique [...]" y alude a los problemas de legibilidad que pueden plantear. Sobre los segundos recuerda que, tanto si se opta por el plural como por el singular, el sentido de la frase siempre ha de ser inclusivo y sugiere que se evite incurrir en incongruencias tales como la femme est un homme comme les autres. Diversas guías lingüísticas de feminización — dirigidas a profesionales de la enseñanza (2006: 3), al personal encargado de redactar textos administrativos y legislativos (2000: 25) o a cualquier persona interesada por las denominaciones de cargos y profesiones (Becquer, 1999: 37-39), por ejemplo— incluyen el recurso al masculino genérico entre sus propuestas, si bien aconsejan un empleo moderado de este procedimiento o insisten en que el enunciado no ha de ser contrario a la lógica ni a la gramática. Y cada vez son más los lingüistas que cuestionan abiertamente los conceptos de masculino genérico, género no marcado o neutro. 
Frente a la Academia francesa, que rechaza los vocablos femenino y masculino y se inclina por el género no marcado por considerar que el marcado segrega, discrimina o, cuando menos, separa o excluye (Rousseau, 1998: 6), se alzan voces para refutar sus razonamientos. Patricia Niedzwiecki (1994: 91-107) opina que tenemos que dejar de seguir normas arbitrarias e ilógicas que generan confusión. Se niega a aceptar que el femenino sea el género marcado porque supondría admitir que se trata de una desviación, de una anomalía y, por lo tanto, quedaría relegado a un segundo plano. Rebate las tesis de quienes se posicionan a favor del neutro en francés y explica la naturaleza del masculino genérico "[...] ou le féminin travesti”.

Como ella, Louise-L. Larivière (2000: 42-65) piensa que la noción de género no marcado es una aberración "[...] puisque c'est le propre du genre d'avoir des marques, féminines et masculines, pour classer les êtres et les choses”; $y$, al referirse a la marca cero, aclara que "[...] c'est une marque virtuelle qui n'a pas pris forme”. Al igual que la autora canadiense Céline Labrosse (2002: 33-38), se erige en defensora de una gramática no sexista. Se opone a la regla que estipula que el femenino se forma a partir del masculino y a la prelación de un género sobre otro. Contrarresta estas normas con diversos argumentos. Esgrime que las marcas del género se describen más fácilmente tomando como base el femenino y no al revés, sobre todo en francés oral; llama la atención sobre las incoherencias semánticas y sintácticas a las que puede dar lugar el seudoneutro y señala los malentendidos que se producen cuando "le générique dégénère". Declara su disconformidad con la aplicación de dicho criterio, dado que restringe el alcance del femenino, y recuerda épocas antiguas en las que no se restaba valor a este género.

\subsection{Tipos de sexismo}

Antes que ella, y también después, otros autores han evidenciado la pervivencia en la lengua francesa de diversos tipos de sexismo, que subsisten en todos los niveles y sin que, en determinados casos, los usuarios sean conscientes de ello. En 1979, Marina Yaguello (185) señalaba: "En effet, pour une large part, le sexisme qui imprègne la langue, comme d'ailleurs les autres manifestations culturelles, telles que les mœurs, lois, etc. n’est pas clairement perçu, car il a été longtemps intériorisé par les locuteurs".

Años más tarde, Benoîte Groult (1984: 27-28) retomaba la idea de que la lengua no es neutra, dado que refleja y alimenta sutilmente prejuicios y estereotipos inculcados desde la infancia. Y Labrosse (18) insistía en 2002 en la influencia del lenguaje en el comportamiento y las percepciones de las personas y cuestionaba los postulados androcéntricos que subyacen en la comunicación lingüística.

Numerosos son los especialistas que, individualmente o en colaboración con profesionales de otras actividades, han puesto de manifiesto usos sexistas gramaticales, léxico-semánticos y discursivos. 


\subsubsection{Gramatical}

En el nivel gramatical, determinados empleos del masculino genérico fomentan la invisibilidad de las mujeres y la refuerzan incongruentemente los casos de concordancia en los que no existe conformidad entre dos palabras, como, por ejemplo, el sustantivo grand-mère -rémora del pasado que todavía no se ha subsanado, dado que sigue sin admitir la variación de género; o el incoherente uso del artículo le para designar a una mujer (madame le ministre, madame le secrétaire d'état, etc.), que, con no pocos esfuerzos, se ha logrado desterrar (Rousseau, 1998: $1-5)$.

\subsubsection{Léxico-semántico}

En el nivel léxico-semántico, tropezamos con varios escollos: los falsos masculinos genéricos, los duales aparentes, los vacíos léxicos, las expresiones estereotipadas, las formas de tratamiento inadecuadas o el obstáculo de las designaciones profesionales, entre otros. Louise-L. Larivière (2000: 42-44) rechaza que homme sea un verdadero genérico:

[...] quand on dit que "homme" est un terme générique qui englobe les espèces "femme" et "homme”, on construit mal l'“arbre” de la structure hiérarchique, qui ne peut contenir un terme (homme) qui soit, à la fois, le tronc (le terme générique) et une branche (un de ses termes spécifiques). Chaque embranchement de l'arbre, à quelque niveau qu'il se situe, doit avoir sa propre dénomination.

Y propone sustituirlo por être humain. Marina Yaguello (1979: 141-173) profundiza en la falta de simetría semántica y hace recaer en los diccionarios gran parte de la responsabilidad de la discriminación lingüística por motivo de sexo. Tras analizar parejas de términos tales como femme/homme, fille/garçon, féminin/masculin, maternel/paternel, etc., concluye que la asimetría deriva de “[...] la péjoration généralisée de tout ce qui sert à qualifier ou à désigner les femmes” en las sociedades patriarcales y que los diccionarios contribuyen a mantenerla dado que sustentan la ideología dominante. Tanto la elección de las palabras y expresiones que figuran en ellos como los componentes denotativo, connotativo y asociativo de las mismas revelan el cariz conservador de este tipo de obras. Benoîte Groult (1984: 31) señala que se sirven a menudo de eufemismos y metáforas que devalúan la feminidad y Céline Labrosse (2002: 13 y 67-68) cuestiona la autoridad que detentan pues:

Que ce soit par l'absence de termes pour désigner des réalités propres aux femmes ou encore par les représentations des femmes qui, lorsqu'elles réussissent à émerger figurent derrière celles de leurs confrères, le déséquilibre qui marque les désignationset les références des unes par rapport aux uns est manifeste.

Se pregunta por qué no recogen locuciones equivalentes a las que constan en la entrada homme: "[...] femme de poids, femme de génie, femme de goût, femme de 
mérite [...]”, etc. O por qué expresiones como rire dans sa barbe no se adaptan a la realidad de las mujeres.

En lo que concierne a las formas de tratamiento, persisten distintos tipos de discriminación. Si bien el apelativo mademoiselle ya no alude al estado civil de la mujer, marca únicamente la diferencia de edad y está en vías de desaparición (Houdebine, 1987: 16) y madame la seguido de la profesión del marido ya no indica la posición social de la esposa (Niedzwiecki, 1994: 43), cuando se casan, las francófonas no sólo pierden su apellido y adoptan el de su cónyuge, sino que en ocasiones llegan a perder su propio nombre y son designadas con el de su consorte o conservan el suyo, pero se las nombra sin apellido. Cierto es que se han promulgado leyes que permiten conservar el apellido de soltera a la mujer casada y trasmitírselo a sus hijos junto al del marido (Yaguello, 1979: 176-177) o de manera exclusiva, como en Québec (Labrosse, 2002: 57); sin embargo, las complicaciones administrativas, la confusión que generan y el peso de las tradiciones disuaden a muchas mujeres. Además, Céline Labrosse (2002: 54-56) comenta los resultados de un estudio suyo sobre los apellidos de los quebequenses que demuestra que la mayoría de los patronímicos (el 81\%) remite al universo masculino. Por otro lado, se empequeñece o encajona a las mujeres cuando se les asigna el nombre propio: un diminutivo (Pierrette, Paulette) o un nombre de flor o que evoca una cualidad moral, acordes con los estereotipos vigentes (Yaguello, 1979: 178-179); o se les atribuye un lenguaje específicamente femenino, afirmación que trasluce prejuicios injustificados que empiezan a desenraizarse (Labrosse, 2002: 101-109).

Por lo que respecta al léxico empleado para referirse a profesiones o cargos desempeñados por mujeres, Niedzwiecki (1994: 70 y 185) defiende la necesidad de adaptar la lengua a la realidad venciendo la resistencia que ofrecen quienes aún se oponen a la visualización de la dimensión femenina porque consideran que la feminización conlleva la pérdida de valor y prestigio. Y recuerda que las designaciones profesionales tienen varias dimensiones - física, lógica, sicológica y moral—, que confieren la identidad a las personas:

[...] le terme professionnel fait apparaître dans l'exercice d'une profession bien plus qu'un simple mot: le costume, l'outillage, l'équipement utilisé, les conditions de travail, la position sociale, la déontologie, la terminologie spécifique à la profession, l'échelle salariale, la position attributive de responsabilités et de devoirs envers les groupes sociaux et professionnels.

También en relación con el ámbito profesional, Louise-L. Larivière (2000: 7078) subraya la diferencia que existe entre los empleos modestos y los puestos de prestigio. Se remonta a la Edad Media para demostrar que, en lo que concierne a los primeros, siempre ha habido apelaciones diferentes para designar a trabajadores de los dos sexos; sin embargo, en lo que toca a los segundos, la discriminación data del siglo XVII. Hace resaltar el machismo lingüístico que todavía persiste aunque se vaya corrigiendo progresivamente. Destaca la desigual percepción del sentido de sustantivos y adjetivos tales como cuisinier/cuisinière, un homme public/ une femme publique, etc.; y el menosprecio que supone referirse o dirigirse a una mujer con un término masculino, algo ilógico y agramatical. 


\subsubsection{Discursivo}

En el discurso aparecen los usos sexistas gramaticales y léxico-semánticos mencionados y es especialmente llamativo el olvido de las mujeres en el modo de construir el mensaje. Numerosos documentos recurren a menudo al masculino genérico - que a veces confunde y molesta a las interesadas; otros, como las ofertas de empleo, se dejan llevar por prejuicios arraigados. A pesar de que existe una nueva sensibilidad a la hora de redactar escritos oficiales "[...] rompant avec une attitude d'indifférence voire d'hostilité [...]" (Chancellerie fédérale, 2000: 3), la llamada ley de economía lingüística suele primar: "[...] la loi du moindre effort, ou loi de l'inertie, (dite encore, en enjolivant les choses, loi de l'économie)" (Niedzwiecki, 1994: 229); y, si no, prevalece la norma de la inteligibilidad de los textos (2006: 1, 2, 9 y 10). Además se dan otras formas de discriminación, tales como la presentación de la mujer, no por sí misma, sino por su relación con su compañero o marido; o el trato desigual, cuando se nombra o identifica a la mujer por su sexo y al hombre por su profesión o su posición social.

1.3. Progresivas modificaciones: diferencias entre países y entre lengua oral y lengua escrita

En las últimas décadas, la lengua francesa ha avanzado por el camino de la igualdad; no obstante, los progresos efectuados no han sido ni rápidos ni homogéneos. Québec es el abanderado de esta reforma: en los años 60 abre el debate. En los 70 y los 80 se involucran las instituciones canadienses: propugnan la eliminación de los estereotipos sexistas y patrocinan la publicación de diversos trabajos relacionados con el tema (Gouvernement de l'Ontario, 2003). A ellas se suman diferentes organizaciones sindicales, municipales, profesionales, etc. Lingüistas, medios de comunicación y ciudadanas y ciudadanos canadienses se implican activamente en la defensa de cambios decisivos, que no tardan en recoger determinados diccionarios quebequenses (Labrosse, 2002: 97 y 117). Suiza es el primer país que, en un documento oficial, propone una alternativa al predominio del género masculino: en Le langage n'est pas neutre (1991), recomienda la aplicación de la regla de proximidad (Labrosse, 2002: 86). En Bélgica se promulga en 1993 un decreto que regula la feminización de las designaciones profesionales en los escritos del sector público y, aunque suscita una fuerte oposición (Rousseau, 1998: 28), la polémica y la resistencia no son tan enconadas como en Francia. Este país “[...] a une lourde tradition d'académisme, de purisme et de contrôle sur la langue" (Yaguello, 1979: 135). La posición que adopta la Academia francesa y su relación con el poder político son diametralmente opuestas a las del Office québécois de la langue française (Gilbert, 2008): dicha corporación pone freno a las iniciativas tomadas por el Gobierno francés en esta materia en 1984 y 1998. Desde su fundación en 1635, se aprecia un marcado contraste entre el francés préacadémique y el post-académique (Yaguello, 1979: 135). A partir de entonces se impone "[...] le tamis prescriptif de conventions très contraignantes [...]" para la 
lengua escrita, que ya no evoluciona al mismo ritmo que la lengua hablada, y se coarta la creatividad de los hablantes. Este distanciamiento se halla en el origen de las divergencias que existen entre ambas en lo relativo a la feminización lingüística. El francés oral va por delante, es un ser vivo, se actualiza, forma el femenino como en el pasado: "La langue pré-classique, pré-académique savait former les féminins dont elle avait besoin [...]" (Groult, 1984: 29); no plantea problemas; encuentra soluciones que pueden ser más o menos acertadas, que se consolidan en algunos países francófonos, pero no en otros; ofrece alternativas, es más proclive a la inclusión igualitaria. Como dice Michèle Bourgoin (1984:58), el hecho de que un femenino no figure en el diccionario no implica que no exista o que no sea posible en la lengua hablada. Y, si dicho uso es aceptable, ¿por qué no emplearlo también por escrito?

\subsection{Escollos y soluciones}

Si nos paramos a pensar en los escollos que hay que salvar para feminizar la lengua francesa, vemos que, por lo general, los obstáculos que encontramos no son de carácter lingüístico o que, si afloran dificultades de este tipo, no son insuperables: si nos servimos de las estructuras morfológicas del francés, podemos solventar favorablemente dichos inconvenientes. Las trabas que todavía quedan por vencer son de índole sociocultural y sicológica y evidencian la resistencia activa o pasiva a introducir cambios. Bien por oposición ideológica, bien por inercia, lo cierto es que se ha puesto freno a la innovación con actitudes inmovilistas. Entre otras, el rechazo a echar mano de la sufijación o la flexión gramatical para crear femeninos de forma natural, la posición de quienes se inclinan por el masculino por considerar que este género valoriza una profesión, la defensa acérrima del statu quo o la aceptación ciega de las normas establecidas han entorpecido la adecuación del francés a la realidad actual. No obstante, en las últimas décadas se ha puesto de relieve la inconsistencia de los argumentos esgrimidos por aquellos que se manifiestan contrarios a llevar a cabo modificaciones.

En relación con la feminización de los sustantivos de profesión o cargo, se han aducido diversas objeciones que pueden rebatirse con facilidad y que se sintetizan en cuatro: las observaciones referidas a la homonimia, la eufonía, la desvalorización y el neutro (Becquer, A. et al., 1999: 30-39). Por lo que respecta a la homonimia, se ha invocado la confusión que puede generar la concurrencia de un sustantivo femenino de oficio y un nombre de objeto (fraiseuse) o que designe el estado civil de esposa de (préfète); pero este riesgo se desvanece dado que el contexto despeja la ambigüedad. Además, los defensores de esta postura olvidan que, en el primer caso, sucede lo mismo con el masculino (avocat); o que, en otras épocas, este fenómeno no planteaba problemas: "L'homonymie ne choquait pas les gens du XVI s. quand ils parlaient d'une femme qui soigne en l'appelant médecine [...]" (1999: $30)$. Y, en cuanto al segundo, han podido comprobar que la sociedad ha cambiado; que colonelle ya no se refiere a la cónyuge del jefe militar que manda un regimiento, sino a la mujer que ostenta dicho grado por mérito propio; que los 
diccionarios hacen constar que dicha acepción (consorte de) ha quedado obsoleta y que tan sólo cabe la posibilidad de escuchar algunos ejemplos en lenguaje familiar y tono irónico.

En lo concerniente al efecto disonante que producen algunos femeninos, bien porque resultan antiestéticos, bien porque suenan desagradablemente, hay que decir que, con el uso, el oído se acostumbra a las palabras que las primeras veces le parecieron malsonantes. Y ocurre lo mismo con los sufijos femeninos rechazados porque pueden interpretarse peyorativamente, ya que, con el tiempo, los hablantes se familiarizan con ellos y estas partículas dejan de tener las connotaciones negativas que les habían atribuido. Tampoco es admisible la utilización del masculino para puestos de prestigio con el pretexto de que el femenino rebaja de categoría a las mujeres que los ejercen (directeur/directrice). El razonamiento de la desvalorización es absurdo. Si la feminización de los sustantivos de oficios menos nobles se ha realizado siempre ¿por qué no brindarían un trato igualitario los nombres de cargos u ocupaciones de rango superior plasmando el progreso profesional de las personas de sexo femenino? ¿Por qué la forma femenina deprecia y la masculina neutraliza la diferencia? Para Else Boel (1984: 36-39), la idea de que el masculino, como género no marcado, expresa la función sólo es válida en abstracto, si no se aplica a un individuo concreto; pero Louise-L. Larivière (2000: 50-54) va más allá al afirmar:

[...] le nom "ministre” ne désigne pas et n’a jamais désigné une fonction. Il désigne [...] une personne qui exerce une fonction, et non la fonction elle-même. Si la langue avait voulu que ces noms désignassent une fonction au lieu d'un titre de personne, les dictionnaires l'auraient consigné comme tel. Ce qui n'est pas le cas.

En detrimento de la forma común para los dos géneros, la tendencia a la diferenciación se ha afianzado en los países francófonos.

Por lo general, las soluciones que se han arbitrado para poner fin al sexismo lingüístico han aumentado la visibilidad de las mujeres. Si bien es cierto que las guías de feminización suelen seguir aconsejando la utilización del masculino genérico cuando favorece la legibilidad de los textos y que también se ha tratado de contrarrestar la supremacía del masculino proponiendo el empleo de términos o grupos de palabras que abarcan los dos géneros (tout être humain, les personnes handicapées, le service de traduction, l'équipe de sauvetage, etc.), progresivamente se extiende el uso de fórmulas no discriminatorias tales como los desdoblamientos, las alternativas gráficas, la alternancia en el orden de precedencia, la creación de expresiones idiomáticas y la redacción de documentos en versión femenina.

\section{5. ¿ ¿Intervención o evolución lingüística?}

La asunción social de la igualdad entre mujeres y hombres, las actuaciones de instituciones públicas y la información obtenida por los hablantes a través de la prensa y las guías editadas han favorecido la implantación de la feminización del 
francés (Dister y Moreau, 2013: 9). En 1979, Marina Yaguello (181-193) se preguntaba:

[...] peut-on ou non agir sur la langue afin de la débarrasser du sexisme qui s’y manifeste? Fautil attendre que les mentalités évoluent, entraînant tout naturellement une évolution de la langue, ou bien faut-il au contraire précéder, forcer cette évolution par une action délibérée? Quelle part prend la langue dans la perpétuation des stéréotypes sexistes?

Y a estos y otros interrogantes respondía con lucidez: "l’action volontariste sur la langue" es limitada ya que los esquemas mentales se resisten a cambiar incluso cuando las estructuras sociales han evolucionado; no obstante, la escuela, las instituciones y los medios de comunicación pueden contribuir activamente a erradicar el sexismo lingüístico con manuales, diccionarios, formularios administrativos o programas de televisión no sexistas. Después, diversos especialistas en la materia han seguido su estela. Se ha insistido en el papel esencial que desempeñan los educadores en la lucha contra los prejuicios sexistas y en la necesidad de realizar intervenciones lingüísticas que modifiquen la forma de pensar de los hablantes (Labrosse, 2002: 21, 72 y 137); asimismo se ha señalado la importancia de resexualizar y feminizar el lenguaje por medio de nuevas prácticas traslativas, que sean transgresoras y creativas y que propicien la reflexión sobre la discriminación por motivo de sexo en las lenguas (Martín Ruano, 2006: 205 y 232).

\section{Actividades formativas}

Nosotros, en apoyo de las iniciativas que se han tomado para evitar un uso discriminatorio de la lengua francesa por razón de sexo, vamos a proponer una serie de actividades formativas encaminadas a favorecer el aprendizaje de los estudiantes universitarios de FLE a fin de que tomen conciencia de los recursos que tiene el francés para hacer frente a las dificultades que encuentran o con las que pudieran tropezar. Luchando contra la desinformación y la inercia, tratamos de reorientar sus hábitos comunicativos y procuramos que desarrollen la denominada competencia existencial (Consejo de Europa, 2002: 103-104) en aras de la igualdad lingüística y social. Nuestra propuesta no pretende ser exhaustiva; tan sólo aporta algunas sugerencias, de entre las cuales se escogerán las más adecuadas para cada grupo o estudiante en particular.

Para empezar habría que activar los conocimientos de la clase sobre el tema haciendo descargar una tormenta de ideas y lanzando preguntas tales como: ¿existe relación entre género y sexo en la lengua francesa?, ¿la forma masculina tiene verdadero carácter genérico?, ¿se da la discriminación sexista en este idioma?, ¿de qué medios dispone para combatirla?, ¿'todos los países francófonos han solventado el problema de la misma manera? o ¿en qué medida el fomento de la feminización lingüística llevado a cabo por diversos organismos y profesionales de diferentes ámbitos ha favorecido la igualdad entre mujeres y hombres?

Seguidamente seleccionaríamos algunas muestras de sustantivos femeninos referidos a oficios, profesiones y cargos, entresacados de la prensa francófona - 
"elle est l'auteur", "la secrétaire d’État américaine” (Le Nouvel Observateur, du 14 au 20 juin 2012: 65 y 12); "directrice de cabinet”, "préfète”, "polarde”, "la rédactrice en chef", "une Premier ministre homosexuelle", "une archevêque”, "une top model” (Le Nouvel Observateur, du 28 juin au 4 juillet 2012: 8, 9, 19, 48 y 74); "écrivaine” (Le Devoir, du 22 juillet 1998); "soldate” (Le Soir, du 2 janvier 2012); "professeure” (Le Figaro, du 16 juillet 2012); "poétesse” (Le Temps, du 13 janvier 2012); "le ravissant mannequin Natalia Vodianova” (Le Nouvel Observateur, du 13 au 19 septembre 2012: 40)—; ejemplos de femeninos masculinizados —sagehomme-, masculinos genéricos sexistas — "La gestation, chez l'homme, dure neuf mois” (Larivière, 2000: 45)—, formas de tratamiento no igualitario mademoiselle—, homónimos —cafetière, médecine—, términos considerados disonantes —-sapeuse-pompière—, pronombres válidos para ambos géneros "eille(s)" (Niedzwiecki, 1994: 112), “illes” (Labrosse, 2002: 80)—, nombres compuestos en los que el adjetivo permanece invariable — grand-mère-, etc. En pareja, las estudiantes y los estudiantes acometerían el estudio de cada caso apoyándose en determinadas recomendaciones bibliográficas y electrónicas: en las guías de feminización de los sustantivos de profesiones, oficios y cargos de Francia y de la Comunidad Francesa de Bélgica y en la página web Langagenonsexiste.ca, entre otras. Y después analizaríamos en una puesta en común los aciertos y los desaciertos y reflexionaríamos sobre las distintas posturas adoptadas al respecto.

A continuación harían búsquedas en diversas obras, documentos y medios de comunicación: la palabra étudiante, en el Dictionnaire historique de la langue française; las acepciones y sinónimos de femme, homme, fille y garçon, en diccionarios de la lengua francesa; y ejemplos de sexismo gramatical, léxicosemántico o discursivo en la prensa, en emisiones de radio y televisión, en textos de la Administración y en materiales didácticos. Con la ayuda de guías de comunicación no sexista, tendrían que presentar alternativas, que serían valoradas por el resto de la clase: opciones gráficas, desdoblamientos, concordancia según la regla de proximidad, alternancia en el orden de precedencia, sustantivos colectivos o abstractos, trato igualitario, etc.

Posteriormente cada estudiante redactaría de forma correcta una oferta de empleo, un titular o un pie de foto para un periódico, un aviso dirigido a las usuarias y los usuarios de una biblioteca o al pasaje de un avión, una fórmula de saludo o despedida para emplearla en la radio o en la televisión, etc., que podrían ser evaluados por los compañeros y compañeras y por el profesor.

Y se prepararía para intervenir en un debate o en un seminario que girarían en torno a la feminización de la lengua francesa, pero que se abrirían a otras lenguas - materna y extranjeras. Esta tarea le permitiría detenerse a pensar en su propio modo de expresarse: en si, conscientemente o sin darse cuenta, hace un uso sexista del lenguaje; y ampliar sus conocimientos sobre los diferentes puntos de vista desde los que se puede abordar la cuestión, dado que previamente tendría que consultar documentos que recogen opiniones distintas, por ejemplo: Médias \& Langage, $\mathrm{n}^{\mathrm{o}}$ 19-20, "La langue française au féminin" (1984); "Madame la Ministre". La féminisation des noms en dix questions (Rousseau, 1998); Féminisation (De Villers, 
2013) o La féminisation: quand l'Académie française et l'Office québécois de la langue française passent à l'attaque (Gilbert, 2008). Tras sopesar los argumentos a favor y en contra de poner en práctica determinadas modificaciones y al comparar las soluciones aplicadas en francés con las adoptadas en español, en inglés o en otros idiomas, podría extraer sus propias conclusiones, posicionarse y, quizás, corregir algunos de sus hábitos lingüísticos.

\section{Conclusión}

Para terminar, nos gustaría añadir que el enfoque que hemos propuesto para abordar este tema nos parece el más apropiado; no obstante, existen otras perspectivas desde las que se puede analizar la cuestión de la feminización lingüística. La controversia persiste. Se ha avanzado mucho en el uso igualitario de la lengua francesa, pero todavía queda camino por recorrer. Impulsando la comunicación no sexista en este idioma, se puede seguir luchando contra la discriminación y la invisibilidad de las mujeres. De este modo, se favorece la renovación del francés y paralelamente evoluciona la mentalidad de quienes lo hablan.

\section{REFERENCIAS BIBLIOGRAFICAS}

Becquer, A. et al., (1999) Femme, j'écris ton nom... Guide d'aide à la féminisation des noms de métiers, titres, grades et fonctions. Paris, La documentation française.

Boel, E., (1984) "Masculin, féminin et rôle des sexes" in Médias \& Langage. $\mathrm{N}^{\text {os }}$ 19-20, pp. 35-39.

Bourgoin, M., (1984) “Les noms de métiers et la dérivation” in Médias \& Langage. $\mathrm{N}^{\mathrm{os}}$ 19-20, pp. 57-59.

Communauté française de Belgique. Service de la langue française, (2006) Mettre au féminin: guide de féminisation des noms de métier, fonction, grade ou titre [En línea]. Disponible en: http://www2.cfwb.be/franca/femini/ [Último acceso el 15 de noviembre de 2013].

Confédération suisse. Chancellerie fédérale, (2000) Guide de formulation non sexiste des textes administratifs et législatifs de la Confédération. Berne.

Consejo de Europa, (2002) Marco común europeo de referencia para las lenguas: aprendizaje, enseñanza, evaluación. Madrid, Anaya, Instituto Cervantes y Ministerio de Educación, Cultura y Deporte.

Dister, A. \& M.-L. Moreau, (2013) "Les politiques linguistiques de la féminisation des noms de profession dans les pays francophones du nord" in Langues et cité. $\mathrm{N}^{0} 24$, pp. 9-10.

Gilbert, S.-M., (2008) La féminisation: quand l'Académie française et l'Office québécois de la langue française passent à l'attaque. Bruxelles, Université Libre de Bruxelles.

Gouvernement de l'Ontario. Service de traduction, (2003) La féminisation au gouvernement de l'Ontario [En línea]. Disponible en: 
http://www.sse.gov.on.ca/mgs/onterm/Documents/Guides/guidefem.htm [Último acceso el 15 de noviembre de 2013].

Grevisse, M. \& A. Goosse, (1986) Le bon usage: grammaire française. ParisGembloux, Duculot.

Groult, B., (1984) "Les mots et les femmes" in Médias \& Langage. $\mathrm{N}^{\mathrm{os}} 19-20$, pp. 27-33.

Houdebine, A.-M., (1987) "Le français au féminin” in La Linguistique. Vol. 23, fasc. 1, pp. 13-34.

Labrosse, C., (2002) Pour une langue française non sexiste. Montréal, Les intouchables.

Labrosse, C., (2005) Langagenonsexiste.ca: pour l'égalité des femmes et des hommes dans la langue [En línea]. Disponible en: http://www.langagenonsexiste.ca/menu.htm [Último acceso el 14 de noviembre de 2013].

Larivière, L.-L., (2000) Pourquoi en finir avec la féminisation linguistique ou à la recherche des mots perdus. Montréal, Boréal.

Le Devoir du 22 juillet 1998 [En línea]. Disponible en: http://www.ledevoir.com/ [Último acceso el 4 de octubre de 2013].

Le Figaro.fr du 16 juillet 2012 [En línea]. Disponible en: http://www.lefigaro.fr/ [Último acceso el 6 de octubre de 2013].

Le Nouvel Observateur du 14 au 20 juin 2012.

Le Nouvel Observateur du 28 juin au 4 juillet 2012.

Le Nouvel Observateur du 13 au 19 septembre 2012.

Le Soir du 2 janvier 2012 [En línea]. Disponible en: http://www.lesoir.be/ [Último acceso el 23 de septiembre de 2013].

Le Temps du 13 janvier 2012 [En línea]. Disponible en: http://www.letemps.ch/ [Último acceso el 23 de septiembre de 2013].

Martín Ruano, M. R., (2006) “Gramática, ideología y traducción: problemas de la transferencia asociados al género gramatical” in Elena, P. \& J. de Kock (eds.), Gramática y traducción. Salamanca, Universidad de Salamanca, pp. 205-237.

Niedzwiecki, P., (1994) Au féminin! Code de féminisation à l'usage de la francophonie. Paris, Nizet.

Ordre des enseignantes et des enseignants de l’Ontario, ( $\dot{2} 2006$ ?) Guide de féminisation. Toronto.

Rey, A., (1998) Dictionnaire historique de la langue française. Paris, Dictionnaires Le Robert.

Rousseau, J., (1998) “Madame la Ministre". La féminisation des noms en dix questions [En línea]. Disponible en: http://www.ciep.fr/chroniq/femi/fem.pdf [Último acceso el 15 de noviembre de 2013].

Villers, M.-É. de, (2013) Féminisation [En línea]. Disponible en: http://www.hec.ca/qualitecomm/chroniques/femini/ [Último acceso el 15 de noviembre de 2013].

Yaguello, M., (1979) Les mots et les femmes. Paris, Payot. 